\title{
STATUS OF THE PEP-II TRANSVERSE FEEDBACK SYSTEMS*
}

\author{
W. Barry, J. Corlett, M. Fahmie, J. Greer, G. Lambertson, C. Pike, Lawrence Berkeley National \\ Laboratory, Berkeley, CA 94720.
}

The feedback systems for controlling transverse coupled-bunch instabilities in both the high and low energy rings of the PEP-II collider are complete and installed on site. Presently, the high energy ring of the collider is beginning commissioning. The commissioning of the high energy ring transverse feedback system is expected to begin sometime in the next few weeks. Design specifications and the basic system concept are reviewed. Selected bench-test results for various system components are discussed including results from the sumsignal orbit offset cancellation electronics.

\section{INTRODUCTION}

The PEP-II B-Factory is a high-luminosity, asymmetric electron-positron collider for studying and determining the origin of $\mathrm{CP}$ violation in the B-meson system $^{1}$. The collider consists of a $9 \mathrm{GeV}$ high-energy storage ring (HER), and a $3.1 \mathrm{GeV}$ low-energy storage ring (LER). Presently, the HER is being commissioned while construction of the LER continues with an expected completion date of April, 1998.

Because of the high average beam currents $(1 \mathrm{~A}$ HER and 2.14 A LER), active feedback systems for controlling longitudinal ${ }^{2}$ and transverse ${ }^{3}$ coupled-bunch instabilities are required. The storage rings are designed to accommodate a large number of bunches, up to 3316 in buckets separated by $2.1 \mathrm{~ns}(476 \mathrm{MHz} \mathrm{RF})$. As a result, a broad and dense spectrum of transverse coupled-bunch modes are driven by the higher-order transverse modes of the RF cavities and the transverse resistive-wall impedance. In order to effectively damp and control growth of these modes, the transverse feedback systems must be broad-band with the capability of providing bunch-bybunch correction. Systems for both the HER and LER which have been modeled after the ALS transverse bunchby-bunch system ${ }^{4}$, have been designed, constructed and installed at the PEP-II sector 4 straights. In this paper, the system concept and specifications are reviewed with emphasis placed on some of the more challenging hardware designs and results.

\section{SYSTEM SPECIFICATIONS}

Nominally, PEP-II will operate with every other bucket filled (238 $\mathrm{MHz}$ bunch rate) and a small ion clearing gap. In this case, the minimum required bandwidth for the feedback system is $119 \mathrm{MHz}$. However,

*Supported by the US Department of Energy under Contract number DE-AC03-76SF00098 (LBL) and DEAC03-76SF00515 (SLAC) the feedback systems, as indicated in table 1 , have been designed to have a bandwidth of $250 \mathrm{MHz}$ in order to accommodate the possibility of operating with every bucket filled. One exception is the stripline kickers which are designed to cover the DC - $119 \mathrm{MHz}$ band because they are more power efficient than shorter versions which would cover the DC - $238 \mathrm{MHz}$ band. These may be replaced with a $238 \mathrm{MHz}$ design, and if needed, more power amplifiers may be added if the $476 \mathrm{MHz}$ bunch-rate becomes a likely operating scenario.

\begin{tabular}{ccc}
\hline \hline Parameter & Description & Value \\
\hline $\mathrm{E}$ & Beam energy & $3.1 \mathrm{GeV}$ \\
$\mathrm{f}_{\mathrm{rf}}$ & RF frequency & $476 \mathrm{MHz}$ \\
$\mathrm{I}_{\mathrm{b}}$ & Average current & $3.0 \mathrm{~A}$ \\
$\mathrm{f}_{\mathrm{O}}$ & Orbit frequency & $136.3 \mathrm{kHz}$ \\
$\beta_{\mathrm{av}}$ & Average $\beta$ & $10 \mathrm{~m}$ \\
$\mathrm{v}_{\mathrm{f}}$ & Fractional tune & 0.9 \\
$\tau_{\mathrm{b}}$ & Bunch spacing & $4.2 \mathrm{~ns}$ \\
$\mathrm{Z}_{\mathrm{rw}}$ & R-wall impedance & $4.85 \mathrm{M} \Omega / \mathrm{m}$ \\
$\alpha_{0}$ & Growth rate of m $=0$ & $3200 \mathrm{sec}^{-1}$ \\
& mode & \\
$\partial \mathrm{V} / \partial \mathrm{x}$ & Req'd feedback gain & $14.6 \mathrm{kV} / \mathrm{mm}$ \\
$\mathrm{R}_{\mathrm{S}}$ & Kicker shunt impedance & $24 \mathrm{k} \Omega$ \\
$\mathrm{P}_{\mathrm{k}}$ & Available kicker power & $240 \mathrm{~W}$ \\
$\mathrm{~V}_{\mathrm{max}}$ & Max. available kick & $3.4 \mathrm{kV}$ \\
$\mathrm{y}_{\mathrm{max}}$ & Max. mode amplitude & $0.23 \mathrm{~mm}$ \\
$\mathrm{~V}_{\mathrm{mode}}$ & Voltage to excite ymax & $71.3 \mathrm{kV}-\mathrm{turn}$ \\
$\Delta \mathrm{f}_{\mathrm{min}}$ & Req'd bandpass & $13.6 \mathrm{kHz}-119 \mathrm{MHz}$ \\
- & Electronics bandpass & $10 \mathrm{kHz}-250 \mathrm{MHz}$ \\
- & Kicker bandpass & $\mathrm{DC}-119 \mathrm{MHz}$ \\
$\sigma_{\mathrm{y}}$ & Vert. beam size & $0.16 \mathrm{~mm}$ \\
- & Req'd dynamic range & $23 \mathrm{~dB}$ \\
- & Actual dynamic range & $42 \mathrm{~dB}$ \\
$\mathrm{y}_{\mathrm{O}}$ & Allowable effective orbit & $1.8 \mathrm{~mm}$ \\
& offset & \\
\hline \hline
\end{tabular}

Table 1. Accelerator and feedback system parameters for assumed worst-case transverse coupled-bunch mode.

The transverse feedback systems are conservatively designed to handle a worst-case scenario which assumes a 3.0 A beam in the LER at a fractional tune of 0.9 (nominal LER current and tune are $2.14 \mathrm{~A}$ and 0.64 respectively). In this case, the fastest growing coupled-bunch mode is the $\mathrm{m}=0$ mode driven by the vertical resistive-wall impedance. Under these conditions, the maximum controllable mode amplitude is $0.23 \mathrm{~mm}$. 
For nominal current and tune, the controllable amplitude is considerably greater. In any case, if the feedback system is operating from the time filling of the storage ring commences, it is unlikely that the modal voltage of 71.3 $\mathrm{kV}$ required to reach the amplitude limit of $0.23 \mathrm{~mm}$ could accumulate apart from interruption of the feedback loop. It should also be noted that simulations indicate ${ }^{5}$ that any expected injection transients are easily damped by the feedback system. This is because the amplitudes of the normal coupled-bunch modes corresponding to the Fourier decomposition of the transient are extremely small. Injection transients can however produce large transient voltages which can saturate the feedback system. For this reason, damping of injection transients usually starts out in saturated mode where a fixed kick is given to the injected bunch until it is damped to a point where proportional feedback takes over.

The required dynamic range is based on the requirement that the amplitude of betatron oscillations be damped to $0.1 \sigma$. For the case in Table 1 , a $23 \mathrm{~dB}$ dynamic range is required to cover mode amplitudes ranging from the maximum of $0.23 \mathrm{~mm}$ down to $0.1 \sigma_{\mathrm{y}}=0.016 \mathrm{~mm}$. The actual dynamic range of the system corresponds to that of an 8 bit D/A, $42 \mathrm{~dB}$. From the required and actual dynamic ranges, the maximum allowable effective orbit offset of $1.8 \mathrm{~mm}$ can be inferred. The effective orbit offset signal depends on the true closed-orbit offset, various electronic gain imbalances, fill pattern, and the effect of the offset rejection circuitry to be described.

\section{FEEDBACK SYSTEM OVERVIEW}

The PEP-II transverse feedback system concept matches quite closely that of the current ALS system and is described in the references. Therefore, only a brief review for orientation purposes is given here with features that are unique to the PEP-II systems pointed out in more detail.

The overall feedback system concept is shown in figure 1. The system utilizes two sets of button pickups separated by some angle in betatron phase for detecting beam moment, I $\Delta \mathrm{x}$. The appropriate vector sum of these is taken to produce a correction signal that is 90 degrees out of phase with respect to beam position at the kickers. This condition results in resistive (no tune shift) damping and is adjustable to allow for changes in tune.

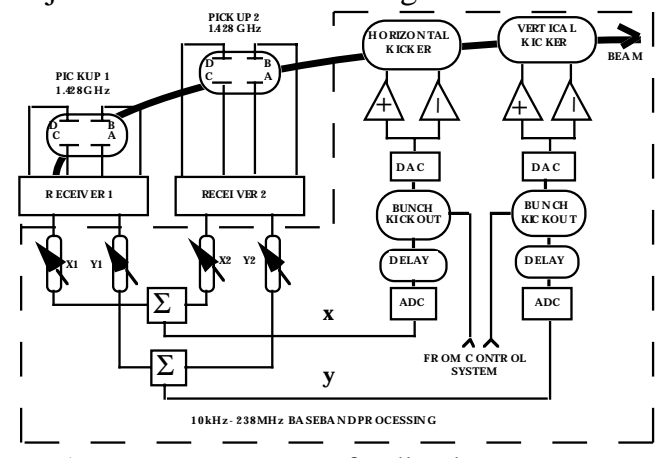

Figure 1. PEP-II transverse feedback system concept.
The beam moment signals are detected at the third harmonic of the RF $(1.428 \mathrm{GHz})$ in order to take advantage of the good sensitivity of the button pickups at this frequency. The signals are demodulated to baseband with heterodyne receivers that also contain orbit offset rejection circuitry. Baseband processing consists of an analog system (shown as $\mathrm{x}$ and $\mathrm{y}$ attenuators) for proportional summing of the two pickup signals and a digital system that provides the necessary pickup-to-kicker timing delay. In addition, the digital electronics features circuitry for changing the sign and gain of the feedback for a given single bunch. This feature is used to trim the charges of single bunches to obtain a uniform fill or kickout an unwanted bunch entirely. Separate horizontal and vertical stripline kickers are used to apply the baseband correction kick to the beam. The kicker electrodes are individually driven with opposite polarities by $120 \mathrm{~W}, 10$ $\mathrm{kHz}-250 \mathrm{MHz}$, solid-state, Class-A, commercial amplifiers. Two components of the PEP-II systems not common to or differing from the present ALS system which presented particularly interesting design and implementation challenges are the receivers with orbit offset cancellation and the digital processors. These components are described in more detail below.

\section{RECEIVER DESIGN}

The PEP-II transverse feedback system receiver design is shown in figure 2. Beam signals induced on each pickup are first bandpass filtered then processed in a microwave monopulse comparator hybrid to produce $\mathrm{x}$ and $\mathrm{y}$ beam moment signals at the $1.428 \mathrm{GHz}$ carrier frequency. Subsequently, the signals are down-converted to baseband using standard heterodyne detection.

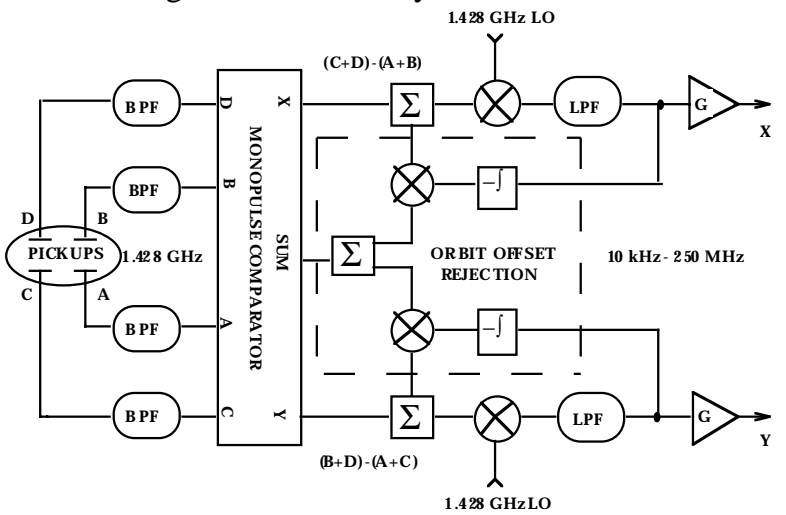

Figure 2. Receiver design.

Of special interest is the orbit offset rejection circuitry in the dashed box. In general, the $\mathrm{x}$ and $\mathrm{y}$ moment signals contain unwanted common -mode components that can saturate the feedback system. This common-mode signal is due to static beam orbit offset and, equivalently, imbalances in the gains of the button pickups and the electronics associated with them. The common-mode or offset signal has frequency components at integer multiples of the orbit frequency only and is spectrally indistinguishable, apart from an overall 
amplitude factor, from the sum signal which is independent of betatron motion. Thus, to remove the common-mode component of the moment signal, some fraction of the sum signal from the comparator is added to or subtracted from it. As shown in figure 2, this process is automated in a feedback loop (which resides in a separate chassis). Figure 3 shows a typical bench measurement of the orbit-offset rejection performance. Here, the large pulse is the baseband receiver output for a simulated offset single bunch and the smaller trace is the bunch signal with the orbit offset rejection turned on. In this case, the rejection is about $20 \mathrm{~dB}$. Because of differences in receiver components, the rejection from unit to unit was found to range from $15 \mathrm{~dB}$ to $26 \mathrm{~dB}$ over a large range of currents and offsets. If one assumes $15 \mathrm{~dB}$ of rejection and $1 \mathrm{~dB}$ of electronic imbalance, the system will handle a beam offset of $8 \mathrm{~mm}$ and still meet the damping specifications in table 1 .

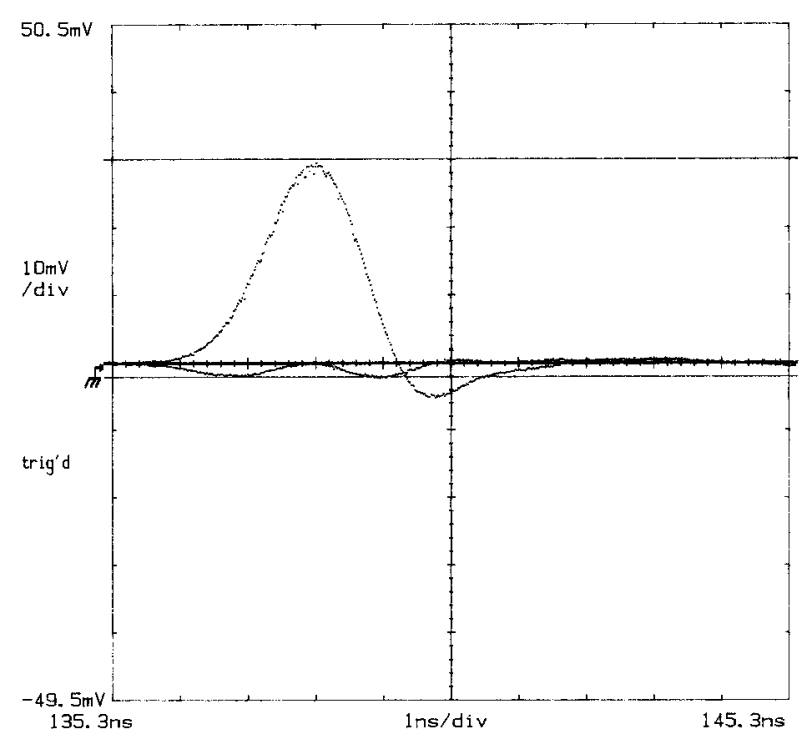

Figure 3. Orbit offset cancellation performance.

\section{DIGITAL COMPONENTS}

The digital components of the system basically consist of a fast analog-to-digital converter, circuitry for blanking or changing the sign of the feedback for a given bunch, a circular memory buffer to provide a pickup-tokicker time delay of up to 8 microseconds, and a fast differential output digital-to-analog converter for driving the pre-amps/power amps. These features provide for the necessary system timing, single-bunch charge trim/kickout, and single-bunch tune measurement without the effects of feedback.

The baseband analog signal is sampled at 476 megasamples per second into 8-bit bi-polar digital form. The sampled data is then directed to a module, which on command, passes the data unaltered or inverts the sign for bunch kickout/trim, or simply blanks the feedback completely. These functions are selectable on a bunch-bybunch basis. In this manner, any number of selected bunches may be kicked out, trimmed, or left uncontrolled simultaneously. The processed values are programmably delayed up to 8 microseconds, in steps of $2.1 \mathrm{nsec}$, by storing them in a circular buffer. The buffer is implemented with a fast ECL RAM array and EClips (tm) ECL logic devices. On alternate memory cycles, the values are written to the RAM array (into incrementing addresses) and then a previously stored value is read out from an offset address. The address offset is programmable and determines the amount of delay applied to the feedback signal. The delayed signal is then converted back into analog format by the fast digital to analog converter.

\section{CONCLUSION}

The broadband transverse feedback systems for the PEP-II storage rings are complete, bench-tested and installed on site. The systems are modeled after the present ALS transverse feedback system which has been running successfully for user operations for almost two years. The design philosophy, as with the ALS system, has been one of simplicity, reliability, and user friendliness. Given our ALS experience, we look forward to the successful commissioning and operation of the PEP-II systems in the upcoming weeks.

\section{ACKNOWLEDGMENTS}

The authors wish to thank the many members of the LBL/SLAC-ALS/PEP-II feedback systems collaboration for many enlightening discussions and useful suggestions.

\section{REFERENCES}

[1] "PEP-II Conceptual Design Report", LBL-PUB-5379, SLAC-418, CALT-68-1869, UCRL-ID-114055, UCIIRPA-93-01, June, 1993.

[2] J. D. Fox, et al., "Observation, Control, and Modal Analysis of Longitudinal Coupled-Bunch Instabilities in the ALS via a Digital Feedback System", AIP Proceedings of the 1996 Beam Instrumentation Workshop, Argonne IL, May 1996.

[3] W. Barry, et al., "Design of the PEP-II Transverse Coupled-Bunch Feedback System", Proceedings of the 1995 IEEE Particle Accelerator Conference, Dallas, TX, May, 1995

[4] W. Barry, et al., "Commissioning and Operation of the ALS Transverse Coupled-Bunch Feedback System", Proceedings of the 1995 IEEE Particle Accelerator Conference, Dallas, TX, May, 1995.

[5] J. Byrd, "Simulations of the PEP-II Coupled-Bunch Feedback Systems", Proceedings of the 1995 IEEE Particle Accelerator Conference, Dallas, TX, May, 1995. 